\title{
Human Papillomavirus (HPV) Concordance in Heterosexual Couples
}

\author{
Lea E. Widdice, M.D. ${ }^{1}$ David J. Breland, M.D., M.P.H. ${ }^{2}$, Janet Jonte, R.N. ${ }^{2}$, Sepideh Farhat, \\ M.S. ${ }^{2}$, Yifei Ma, Ph.D. ${ }^{2}$, Anthony C. Leonard, Ph.D. ${ }^{3}$, and Anna-Barbara Moscicki, M.D. ${ }^{2}$ \\ ${ }^{1}$ Division of Adolescent Medicine, Cincinnati Children's Research Foundation, University of \\ Cincinnati \\ 2 Division of Adolescent Medicine, University of California, San Francisco \\ ${ }^{3}$ Department of Public Health Sciences, University of Cincinnati
}

\section{Abstract}

Purpose-Few studies have examined the relationships between sexual or hygienic behaviors and HPV transmission. Our objectives were to (1) describe HPV concordance between the anogenital, oral and palmar areas of monogamous, heterosexual couples and (2) determine sexual behaviors, hygienic practices, sexual histories and characteristics associated with HPV anogenital concordance.

Methods-Couples were recruited from women who developed an incident HPV infection while enrolled in a longitudinal HPV natural history study that recruited from 2 family-planning clinics. Men were their monogamous partners of at least three months. Samples were tested for HPV-DNA of 37 high- and low-risk genotypes. Questionnaires completed privately assessed health, sexual, hygienic history and behaviors.

Results—25 couples enrolled between February 2006 and July 2007; none had received HPV vaccine. The average age was 25 years (SD 6) for men and 23 years (SD 3) for women. HPV-84 was the most commonly shared HPV type in the anogenital and palmar areas. HPV-16 was the only shared oral-HPV type. $68 \%$ of couples had type-specific anogenital concordance. Receiving finger-anal sex $(\mathrm{p}=.05)$, sharing towels $(\mathrm{p}=.04)$, longer time since last intercourse $(\mathrm{p}=.03$ women and .02 men), and men washing their genitals after sex $(\mathrm{p}=.03)$ were associated with decreased likelihood of concordance. Persistence of incident HPV types in women was associated with HPV in men ( $\mathrm{p}=$. 002).

Conclusions-Our findings show that certain hygienic and sexual behaviors are associated with anogenital concordance between healthy, monogamous, heterosexual couples. Future studies are needed to see if these detections reflect contamination, transient or established infections.

\section{Keywords}

Human Papillomavirus; male; concordance; hygiene; sexual behaviors; cutaneous; oral; genital; transmission; persistence

\footnotetext{
Corresponding Author: Lea E. Widdice, Division of Adolescent Medicine, Cincinnati Children's Hospital Medical Center, 3333 Burnet Ave MLC 4000, Cincinnati, OH 45229, Telephone: 513636 2970, Fax: 513636 1129, lea.widdice@cchcm.org.

Publisher's Disclaimer: This is a PDF file of an unedited manuscript that has been accepted for publication. As a service to our customers we are providing this early version of the manuscript. The manuscript will undergo copyediting, typesetting, and review of the resulting proof before it is published in its final citable form. Please note that during the production process errors may be discovered which could affect the content, and all legal disclaimers that apply to the journal pertain.
} 
Human Papillomavirus (HPV) is recognized as the most common sexually transmitted infection in men and women. Early studies of couples showed low concordance of HPV infection between sexual partner's genital areas, ranging from $2 \%$ to $40 \%$ [1-7]. Although recent data, with improved HPV-DNA detection methods and more standard sampling techniques, suggest higher rates of concordance, they remain well below $100 \%$ with rates ranging from $37 \%$ to $68 \%$ [8-10].

The determinants of concordance between sexual couples are poorly understood and few factors have been shown to be associated with concordance in healthy couples. Higher HPV viral loads may be associated with an increase in concordance, but this finding is inconsistent. Direct comparison of these studies is difficult since reports lack information on the time between sampling of partners $[7,10,11]$. Additionally, faster clearance in one partner may explain lack of concordance when couples are examined cross-sectionally; longitudinal studies suggest men clear genital HPV faster than women $[12,13]$. Few studies have examined behavioral factors commonly attributed to the spread of infectious agents such as specific sexual practices and sharing of hygiene products.

Certainly, one of the factors potentially affecting concordance is the type of sexual relationship. Partners in transient relationships may have less time to pass an infection whereas monogamous partners who have sex frequently may transmit HPV more successfully if an infection is introduced into the relationship by pre-existing or latent infections. Another factor may be the stage of HPV infection. Many studies have focused on women with later stages of HPV infection including women with invasive cervical cancer [5], which may be less likely to shed infectious particles, and women with abnormal cytology $[2,4,6,8,9]$, which is associated with higher viral loads. Also, studies of concordance have focused primarily on the genital area. It is known that anogenital HPV genotypes can be detected from non-anogenital sites such as the oral cavity and surface of the hand $[14,15]$. To date, little is known about the concordance between couples when multiple sites are considered.

The objectives of this study were to (1) describe HPV concordance between the anogenital, oral and palmar areas of monogamous, heterosexual couples and (2) to determine sexual behaviors, hygienic practices, sexual histories and characteristics of individuals and couples that are associated with HPV type-specific anogenital concordance between sexual couples.

\section{Methods}

\section{Participants}

Female partners were recruited from participants of an on-going natural history study of HPV that enrolled women aged 13 to 21 years who were attending 1 of 2 family planning clinics from 1990 to 1994 and 2000 to 2004 in California. Participants have a study-visit every four months during which demographic and behavioral information are obtained. Cervical HPV testing, cytology and colposcopy are performed [13,16-18].

Women were identified for recruitment into the couples' study if they had an incident cervical HPV type detected at the most recent natural history study visit. Incident cervical HPV type was defined as a cervical HPV type detected that was not detected on the previous visit. These women were eligible for recruitment if they were in a monogamous, heterosexual relationship of at least three months duration, not pregnant, had normal cytology and had no current history of genital warts.

Women's eligibility was determined by review of study chart and confirmed in conversation with the women by study staff. Each eligible, interested woman was asked to obtain permission from her partner for the study staff to contact him. The study, including confidentiality and 
eligibility, were explained to men privately via telephone. Eligibility criteria for men included self-reported monogamy for three months or longer, not having genital warts and no use of medication in the genital area at the time of enrollment. Men and women were allowed to opt out at any point without stating a reason. All subjects were 18 years or older and provided informed consent. The Institutional Review Boards of University of California, San Francisco; San Francisco State University; University of Cincinnati; and Cincinnati Children's Hospital Medical Center approved this study.

\section{Procedures}

Couple's visits occurred within eight weeks of the woman's visit at which the incident cervical HPV was detected. Partners were sampled for HPV on the same day. Each participant completed a self-administered paper-pencil questionnaire privately and separately from his or her partner. Information collected by questionnaire included: participant's demographic characteristics, health history, sexual history and recent sexual and hygienic behaviors. No information was shared with partners.

A trained clinician collected samples from each participant in private. Each HPV-DNA sample was collected using separate supplies, including gloves. All swabs were sterile Dacron. In women, a swab was placed $2 \mathrm{~cm}$ into the anal canal and rotated $360^{\circ}$ conically three times, a swab was used to scrape the vulvar area (introital, labia minor) three times on each side and a swab was used to scrape the vaginal walls three times. The cervix was sampled using a $5 \mathrm{~mL}$, normal-saline, cervicovaginal lavage. Cervical cytology was obtained after HPV-DNA samples using a cytobrush and spatula. Cells were immediately transferred onto a glass slide and placed into methanol $[16,18,19]$. In men, the glans, including corona sulcus; shaft; inner foreskin, if applicable; scrotum and perianal area were sampled separately using textured papers ( $2 \mathrm{~cm}$ by $3 \mathrm{~cm} 600 \mathrm{~A}$-grit Wetordry Tri-M-ite) to exfoliate each site prior to wiping with normal-saline moistened swabs. In men and women, the palmar surface, including fingers, of the dominant hand was sampled using one textured paper and moistened swab per subject. Oral samples in men and women were collected from the buccal mucosa and tongue with a cytobrush [20,21]. Swabs and cytobrushes were immediately placed into separate vials of sample transport media (QIAGEN Inc., Valencia, CA). Textured papers were placed into separate, dry cryovials. Specimens were stored and tested in Dr. Moscicki's laboratory.

HPV-DNA detection used Linear Array HPV Genome Typing Test ${ }^{\mathrm{TM}}$ (Roche Molecular Systems, Pleasanton CA) [22,23] for 37 anogenital genotypes $(6,11,16,18,26,31,33,35$, $39,40,42,45,51-59,61,62,64,66-73,81-84$, IS39 and CP6108) per manufacturer's instructions. Human $\beta$-globin DNA served as a positive control. Samples were prepared using QIAamp MinElute Media Kit (QIAGEN Inc. Valencia, CA). One pathologist read all cytology.

\section{Analyses and Statistical Methods}

Results from individual samples from the anogenital area (vulva, vagina, cervical, and anal samples in women and glans, shaft, foreskin, if applicable, scrotum, and perianal areas in men) were combined for analysis after HPV-DNA testing since the sample size precluded analysis of individual sites. Samples with negative $\beta$-globin and HPV-DNA signals were considered inadequate and excluded from analysis. By definition, all women in the study were HPV positive within eight weeks of the baseline visit of this transmission study. Therefore, typespecific concordance was defined as the co-occurrence of an HPV type in both partners (positive concordance) or as the absence of all HPV types in both partners (negative concordance). Persistence was defined as redetection of the incident, cervical HPV type in the woman's anogenital area at the couple's visit. 
Two-tailed p-values for comparisons between men and women were obtained from sign-rank tests for interval variables and McNemar's tests for dichotomous variables. Measures of agreement within couples are Spearman rank correlations for interval variables and Kappas for dichotomous variables. All p-values associated with tests of association between typespecific concordance and any predictors are mid p-values from a conditional exact test using the "score" method [24,25]; odds ratios (ORs) are exact with exact mid-p confidence limits, each was obtained from SAS Proc Logistic. The use of conditional exact tests and mid-p values was chosen a priori because of our small sample size [24,25]. Mid-p values for $2 \times 2$ tables are the Fisher's exact p-values (sum of the probabilities of the observed table under the null hypothesis plus those of all possible tables as probable or less probable than the observed table) minus $1 / 2$ the probability of the observed table. Note that mid-p significance tests share with Fisher's test the possibility of attaining a two-tailed value less than .05 while the OR's exact $95 \%$ confidence interval (CI) includes 1. This is because the 95\% CI for the OR is centered in the sense that $2.5 \%$ of the probability is beyond each end of the CI. The test of independence, however, may be uncentered in that the one-tailed p-value is not necessarily $1 / 2$ the two-tailed value. Other comparisons of independent proportions were tested using two-tailed Fisher's exact tests. The study alpha was .05 , two-tailed and p-values were not adjusted for multiple comparisons. The intention of these analyses was to generate hypotheses regarding subject characteristics and behaviors and therefore did not include multiple infections or oncologic risk of HPV types.

\section{Results}

\section{Subject Characteristics and HPV Detection}

Twenty-five couples were enrolled between February 2006 and July 2007. No subjects had received HPV vaccination. Forty-four of the 79 women (56\%) eligible for the study provided permission to contact their partners. Eight men could not be contacted and three were ineligible, leaving 33 (75\% of recruited men) eligible for enrollment. Eight couples did not enroll. Of the enrolled couples, twelve lived together. Rates of reported sexually transmitted infections, smoking, alcohol and substance use for men and women are summarized in Table 1. Few couples used condoms and the majority of men (64\%) were circumcised. The average age of men was 25 years (S.D. 6 years). The average age of women was 23 years (S.D. 3 years) ( $p=$ 001). Men reported a longer period of time since the couple last had intercourse than did women $(\mathrm{p}=.02)$. Although men reported the first time the couple had sex to be more recent than did women, the difference was not significant (Table 1). Agreement of reported characteristics and behaviors within couples is shown in Table 1.

Table 2 shows the distribution of anogenital, palmar and oral HPV types at the couple's visit and the incident types detected in women prior to the couple's visit. Each partner had adequate samples from their palmar site and anogenital area. One woman had an inadequate oral sample. The most common types detected in the anogenital area of men were HPV 84 (24\% of men), $16(20 \%), 52,53,39(16 \%)$. The most common types of HPV detected in the anogenital area of women were HPV 84 (24\% of women), 52 and 53 (20\%), and 39 and $16(16 \%)$.

\section{Type-Specific Anogenital Concordance and Persistence of the Incident HPV Type}

Type-specific anogenital concordance occurred in $68 \%$ of the couples $(n=17)$. Three couples had negative concordance, 14 couples had positive concordance for 1 to 5 types (Table 3 ). The most commonly shared type between partners' anogenital areas was 84 , found in five couples (20\%) followed by 39,52 , and 62 , found in three couples each (12\%).

Type-specific concordance at the palmar area was $68 \%$ ( $\mathrm{n}=17$ couples). Of these, 13 couples (52\%) were HPV negative and four couples (16\%) shared at least one HPV type; two couples 
shared one type and two couples shared two types. The most commonly shared type between partners' palmar area was 84 , found in two couples (8\%). Oral HPV was rarely detected; twenty-one couples $(87.5 \%)$ were negative for oral HPV. Only one couple had both partners with oral HPV; they shared type 16.

Persistence of the incident HPV type occurred in 18 women (72\%). Men were more likely to be HPV positive for any HPV type in their anogenital area if their partner had persistence of the incident HPV type. Specifically, of the 18 male partners of women with persistence, 17 (94\%) were HPV positive. Of the 7 male partners of women without persistence, 2 (29\%) were HPV positive ( $\mathrm{p}=.002)$. Also, there was a trend toward an association between detection of the woman's incident HPV type in the male partner's anogenital area when the woman had persistence of the incident HPV type. Specifically, of the 18 male partners of women with persistence, $11(61 \%)$ were positive for the woman's incident HPV type. Of the 7 male partners of women without persistence, only $1(14 \%)$ was positive for his partner's incident HPV type $(\mathrm{p}=.07)$.

\section{Shared HPV between partners' non-anogenital and anogenital areas}

Detection of the same HPV types in one partner's palmar and the other partner's anogenital area occurred in 11 couples (44\%). The most common HPV type shared between the palmar area of one partner (either male or female) and the anogenital area of the other partner was type 84 followed by types 51, 39 and 52. Of the seven couples with one or both partners negative for HPV in the anogenital area, no couples had HPV detected in their palmar area. Of the 18 couples with both partners positive for HPV in their anogenital areas, 66\% $(n=12)$ had HPV detected in their palmar areas $(\mathrm{p}=.005)$.

The couple with type-specific concordance at the oral area for HPV type 16 did not have type 16 found in either partner's anogenital area. Of the two couples in which HPV was detected in one partner's oral area, one couple had the same HPV type detected in the other partner's anogenital area. Although not statistically significant, oral HPV was detected only in couples in which both partners were positive for HPV in the anogenital area compared to couples in which one or both partners were negative for anogenital HPV $(\mathrm{p}=.53)$.

\section{Risk Factors Associated with Type-Specific Anogenital Concordance}

Couples were less likely to have type-specific anogenital concordance the longer the time (in hours) between having sex and being sampled for HPV (OR= .98, 95\% CI: .95-1.0, p=.03 for women and OR=.98, 95\% CI: .96-1.0, $\mathrm{p}=.02$ for men). Concordance was less likely if the man reported washing his genital area between having intercourse and being sampled for HPV $(\mathrm{OR}=.16,95 \% \mathrm{CI}: 0-1.0, \mathrm{p}=.03)$ or if the woman reported receiving finger-anal sex $(\mathrm{OR}=.12$, $95 \% \mathrm{CI}: .00-1.42, \mathrm{p}=.05)$, sharing a towel $(\mathrm{OR}=.15,95 \% \mathrm{CI}$. .02-1.1, $\mathrm{p}=.04)$ or sharing a razor $(\mathrm{OR}=.19,95 \% \mathrm{CI}=0-1.6, \mathrm{p}=.05)$ with her partner in the past week. Table 4 shows ORs for selfreported behaviors and health histories from male and female partners associated with typespecific anogenital concordance at a p-value of .10 or less. Factors including male circumcision; duration of monogamy; years sexually active; frequency of vaginal intercourse; the number of days between detection of the incident HPV type and the couple's visit; and other variables listed in Table 1 were not associated with concordance.

\section{Discussion}

This is the first study to our knowledge that examines specific hygienic factors associated with HPV concordance in heterosexual couples. Our study is also unique in that it looked at concordance when the female partner had a recent history of incident HPV infection and normal cytology. Although the number of couples was small, we were able to assess detailed histories 
of specific sexual and hygiene-related behaviors. We believe several of our findings are worth discussion.

To our surprise, we found several relationships between hygienic habits and discordance. Specifically, women who reported sharing a razor or towel with their partners had an increased chance that they would be discordant. This finding appears contradictory to assumptions about HPV transmission; however, very little is actually known about factors that influence HPV detection patterns in couples. We hypothesize that in this case, shared razors and towels pass exfoliated cells infected with HPV DNA from one partner to the other. These exfoliated cells likely come from extra-genital sites (e.g. thighs, pubic hair, etc.) not sampled in the partner. Detection of HPV from the exfoliated cells likely reflects contamination (i.e., the viral DNA is detected because of the infected cellular debris), not an established infection. The detection of HPV from these sloughed cells versus established infections is likely to result in discordance. In this scenario the virus has not entered the subject's basal cell layer and established active replication, rather the HPV-DNA PCR test detects the HPV DNA from the partner's squamous cell. The possibility of contamination by HPV passed between partners was underscored by our finding that men who washed their genitals after sex were more likely to be discordant. Cleaning the genital area after sex may reduce the infectivity of men.

Other findings from our study support the notions that cellular debris with HPV infection or rapidly cleared infections have a role in determining concordance in cross-sectional sampling. The shorter time since a couple had sex, the more likely the same HPV type would be found in both partners. This finding is consistent with the trend toward association found by Baken, et al. [1] in whose study a shorter number of days since last intercourse was weakly associated with increased chance of anogenital concordance between sexual couples. This suggests that either HPV detected in one partner may be simply a contaminant from the other partner that does not result in prolonged infection or that the natural history of HPV infection in men is different than in women with men clearing infections faster than women $[12,13]$.

The type of sexual activity also appeared to affect concordance. Finger-anal sex in women enhanced anogenital discordance. Our finding may indicate that this particular act results in a higher chance of contamination, and thus discordance, of one of the partners. On the other hand, anal HPV infections, in women particularly, have been shown to clear more rapidly than cervical infections [26].

The notion that many HPV-DNA detections are not established infections is underscored by the finding that men who were partners to women with persistent HPV infection were more likely to have HPV detected. Whereas men whose partners did not have persistence of the incident HPV type were unlikely to have HPV detected.

The rate of almost 70\% HPV type-specific concordance is higher than rates reported by others when using the same definition of concordance. In Italy, Benevolo et al. showed a 30.9\% concordance rate. In this study couples reported at least 12 months of monogamy and the women had either a current or past history of CIN or HPV [8]. Baken et al. found a concordance rate of $37.8 \%$ in couples from the United States who were recruited from STD clinic regardless of history of monogamy [1]. In another study of Dutch couples in which the woman had prevalent CIN lesions and recruited without regard to monogamy $39.8 \%$ were concordant [3]. We believe our higher rate of concordance was partly due to our selection of women with known, recent, incident infection and normal cytology. The higher rate of concordance suggests that HPV may be rapidly transmitted shortly after infection. In contrast, partners of women with CIN, who show lower rates of concordance, may have developed an immune response and cleared the HPV infection by the time the male partner was tested. 
Our rate of $8 \%$ oral HPV in men and women is similar to pervious studies using oral rinse sampling that reported rates of 5\% to 8\% [27-29] and lower than a study using cytobrushes to sample Finnish spouses which showed a prevalence of $16 \%$ in women and $18 \%$ in men [30]. Little comparable data are available for HPV detection in the hand. It is interesting to note in this study, HPV was never found in a person's palmar area if the anogenital area was negative. This suggests to us that HPV detected in the hand is primarily a contaminant and not an established infection. Future studies should include HPV testing of the hand in order to determine whether it is a meaningful mode of transmission [15].

Limitations of this study include a small sample size. We attempted to narrow our search by using restrictive eligibility criteria in order to decrease baseline variability of the subjects and by using an a priori analysis plan using statistical methods appropriate for small sample sizes.

Regardless, reported associations should be considered suggestive and appropriate for consideration in future studies. Although monogamy was part of the eligibility criteria and reported by all participants, we acknowledge that self-reports may not reflect the true rates despite our efforts to ensure confidentiality and privacy to enhance truthfulness. Additionally, we required three months or longer of monogamy. Winer et al. [31] found that exposure to a new partner up to eight months prior to HPV sampling was associated with an incident infection. Therefore, the incident infections in the women may have been from contact with other partners beyond the three months. Determination of associations between oral or palmar HPV detection and specific behaviors, such as deep kissing and hand holding, is limited by a lack of information collected.

This study included women with previous HPV exposure. Concordance rates in couples with HPV-naïve female partners may be different since HPV-naïve women will not have developed a cell-mediated immune response to the previously cleared HPV infections. This would result in a longer time of persistence before clearance, thus increasing the chance of concordance. Although we identified incident cervical HPV types in women occurring prior to the couple's visit, the rate of prior anogenital HPV infections is unknown. A longitudinal study is necessary to examine the role of persistent and latent infections on transmission.

In summary, recent sexual behaviors and hygienic habits show a pattern of association with concordance between sexual partners that suggests HPV-DNA detection on cross-sectional sampling may be due to contamination. The transient nature of recent, incident HPV infections is underscored by the fact that incident HPV types were no longer detected in nearly one-third of the women after eight weeks. Longitudinal studies will be critical to elucidate the apparent importance of transient HPV infections. Our data underscore the importance in examining recent sexual and hygienic behaviors in studies of concordance.

\section{Acknowledgments}

Evelyn Hanson, Mia Gutfreund, Julie Jay and Susanna Benningfield for input on study design and data collection, Cheryl Godwin de Medina for recruitment and retention, Lisa Clayton and Annie Wells for data management, Yanhong Lu for laboratory testing and data entry. Tere Darragh, MD for cytology evaluation. I have listed everyone who contributed significantly to this research.

\section{Sources of Support:}

Sources of funding include: National Cancer Institute R37 CA51323, Maternal and Child Health Bureau T71MC00003 and U45MC0023, National Institute of Health/ORWH 1K12 HD051953-01, American Cancer Society \#92-026-12, these studies were carried out in part in the Pediatric Clinical Research Center, Moffitt Hospital, University of California San Francisco with funds provided by the National Center for Research Resources, 5 M01 RR-01271, U.S. Public Health Service; supported in part by a research grant from the Investigator-Initiated Studies Program of Merck $\&$ Co., Inc.. The opinions expressed in this paper are those of the authors and do not necessarily represent those of Merck \& Co., Inc. 


\section{References}

1. Baken LA, Koutsky LA, Kuypers J, et al. Genital human papillomavirus infection among male and female sex partners: prevalence and type-specific concordance. J Infect Dis 1995 Feb;171(2):429_ 432. [PubMed: 7844382]

2. Strand A, Rylander E, Wilander E, et al. HPV infection in male partners of women with squamous intraepithelial neoplasia and/or high-risk HPV. Acta Derm Venereol 1995 Jul;75(4):312-316. [PubMed: 8578958]

3. Bleeker MC, Hogewoning CJ, Berkhof J, et al. Concordance of specific human papillomavirus types in sex partners is more prevalent than would be expected by chance and is associated with increased viral loads. Clin Infect Dis 2005 Sep 1;41(5):612-620. [PubMed: 16080082]

4. Hippelainen MI, Yliskoski M, Syrjanen S, et al. Low concordance of genital human papillomavirus (HPV) lesions and viral types in HPV-infected women and their male sexual partners. Sex Transm Dis 1994 Mar-Apr;21(2):76-82. [PubMed: 9071416]

5. Franceschi S, Castellsague X, Dal Maso L, et al. Prevalence and determinants of human papillomavirus genital infection in men. Br J Cancer 2002 Mar 4;86(5):705-711. [PubMed: 11875730]

6. Rosenblatt C, Lucon AM, Pereyra EA, et al. HPV prevalence among partners of women with cervical intraepithelial neoplasia. Int J Gynaecol Obstet 2004 Feb;84(2):156-161. [PubMed: 14871518]

7. Nicolau SM, Camargo CG, Stavale JN, et al. Human papillomavirus DNA detection in male sexual partners of women with genital human papillomavirus infection. Urology 2005 Feb;65(2):251-255. [PubMed: 15708032]

8. Benevolo M, Mottolese M, Marandino F, et al. HPV genotypes concordance between sex partners. J Exp Clin Cancer Res 2007 Dec;26(4):609-612. [PubMed: 18365561]

9. Giovannelli L, Bellavia C, Capra G, et al. HPV group- and type-specific concordance in HPV infected sexual couples. J Med Virol 2007 Dec;79(12):1882-1888. [PubMed: 17935193]

10. Mbulawa ZZ, Coetzee D, Marais DJ, et al. Genital human papillomavirus prevalence and human papillomavirus concordance in heterosexual couples are positively associated with human immunodeficiency virus coinfection. J Infect Dis 2009 May 15;199(10):1514-1524. [PubMed: 19392625]

11. Bleeker MC, Berkhof J, Hogewoning CJ, et al. HPV type concordance in sexual couples determines the effect of condoms on regression of flat penile lesions. Br J Cancer 2005 Apr 25;92(8):1388-1392. [PubMed: 15812547]

12. Lu B, Wu Y, Nielson CM, et al. Factors associated with acquisition and clearance of human papillomavirus infection in a cohort of US men: a prospective study. J Infect Dis 2009 Feb 1;199(3): 362-371. [PubMed: 19133808]

13. Moscicki AB, Shiboski S, Broering J, et al. The natural history of human papillomavirus infection as measured by repeated DNA testing in adolescent and young women. J Pediatr 1998 Feb;132(2):277284. [PubMed: 9506641]

14. Sonnex C, Strauss S, Gray JJ. Detection of human papillomavirus DNA on the fingers of patients with genital warts. Sex Transm Infect 1999 Oct;75(5):317-319. [PubMed: 10616355]

15. Hernandez BY, Wilkens LR, Zhu X, et al. Transmission of human papillomavirus in heterosexual couples. Emerg Infect Dis 2008 Jun;14(6):888-894. [PubMed: 18507898]

16. Scott ME, Ma Y, Farhat S, et al. Covariates of cervical cytokine mRNA expression by real-time PCR in adolescents and young women: effects of Chlamydia trachomatis infection, hormonal contraception, and smoking. J Clin Immunol 2006 May;26(3):222-232. [PubMed: 16783462]

17. Moscicki AB, Palefsky J, Gonzales J, et al. Human papillomavirus infection in sexually active adolescent females: prevalence and risk factors. Pediatr Res 1990 Nov;28(5):507-513. [PubMed: 2175024]

18. Farhat S, Nakagawa M, Moscicki AB. Cell-mediated immune responses to human papillomavirus 16 E6 and E7 antigens as measured by interferon gamma enzyme-linked immunospot in women with cleared or persistent human papillomavirus infection. Int J Gynecol Cancer 2009 May;19(4):508512. [PubMed: 19509544] 
19. Moscicki AB, Hills N, Shiboski S, et al. Risks for incident human papillomavirus infection and lowgrade squamous intraepithelial lesion development in young females. JAMA 2001 Jun 20;285(23): 2995-3002. [PubMed: 11410098]

20. Weaver, BA. Male HPV Specimen Collection Procedure [Instructional Video]. Hawaii: Cancer Research Center, University of Hawaii; 2004.

21. Weaver BA, Feng Q, Holmes KK, et al. Evaluation of genital sites and sampling techniques for detection of human papillomavirus DNA in men. J Infect Dis 2004 Feb 15;189(4):677-685. [PubMed: 14767822]

22. Castle PE, Gravitt PE, Solomon D, et al. Comparison of linear array and line blot assay for detection of human papillomavirus and diagnosis of cervical precancer and cancer in the atypical squamous cell of undetermined significance and low-grade squamous intraepithelial lesion triage study. J Clin Microbiol 2008;46(1):109-117. [PubMed: 17989194]

23. Gravitt PE, Peyton CL, Alessi TQ, et al. Improved amplification of genital human papillomaviruses. J Clin Microbiol 2000;38:357-361. [PubMed: 10618116]

24. Agresti A. A survey of exact inference for contingency tables (with discussion). Statistical Science 1992;7:131-177.

25. Lancaster HO. Significance tests in discrete distributions. Journal of the American Statistical Association 1961;56:233-234.

26. Shvetsov YB, Hernandez BY, McDuffie K, et al. Duration and clearance of anal human papillomavirus (HPV) infection among women: the Hawaii HPV cohort study. Clin Infect Dis 2009 Mar 1;48(5):536-546. [PubMed: 19191636]

27. Smith EM, Swarnavel S, Ritchie JM, et al. Prevalence of human papillomavirus in the oral cavity/ oropharynx in a large population of children and adolescents. Pediatr Infect Dis J 2007 Sep;26(9): 836-840. [PubMed: 17721381]

28. Summersgill KF, Smith EM, Levy BT, et al. Human papillomavirus in the oral cavities of children and adolescents. Oral Surg Oral Med Oral Pathol Oral Radiol Endod 2001 Jan;91(1):62-69. [PubMed: 11174573]

29. Kreimer AR, Alberg AJ, Daniel R, et al. Oral human papillomavirus infection in adults is associated with sexual behavior and HIV serostatus. J Infect Dis 2004 Feb 15;189(4):686-698. [PubMed: 14767823]

30. Rintala M, Grenman S, Puranen M, et al. Natural history of oral papillomavirus infections in spouses: a prospective Finnish HPV Family Study. J Clin Virol 2006 Jan;35(1):89-94. [PubMed: 16112613]

31. Winer RL, Lee SK, Hughes JP, et al. Genital human papillomavirus infection: incidence and risk factors in a cohort of female university students. Am J Epidemiol 2003 Feb 1;157(3):218-226. [PubMed: 12543621] 


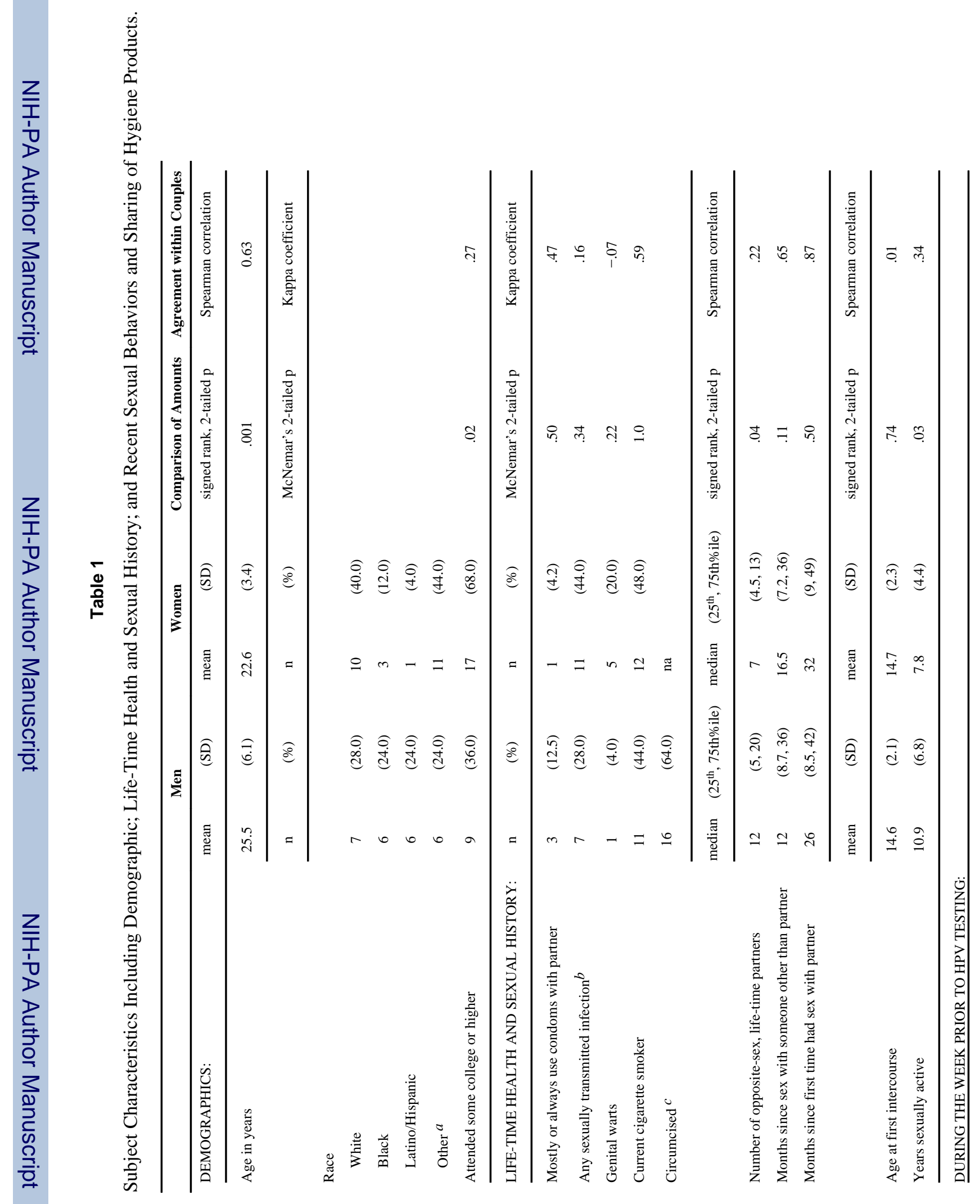




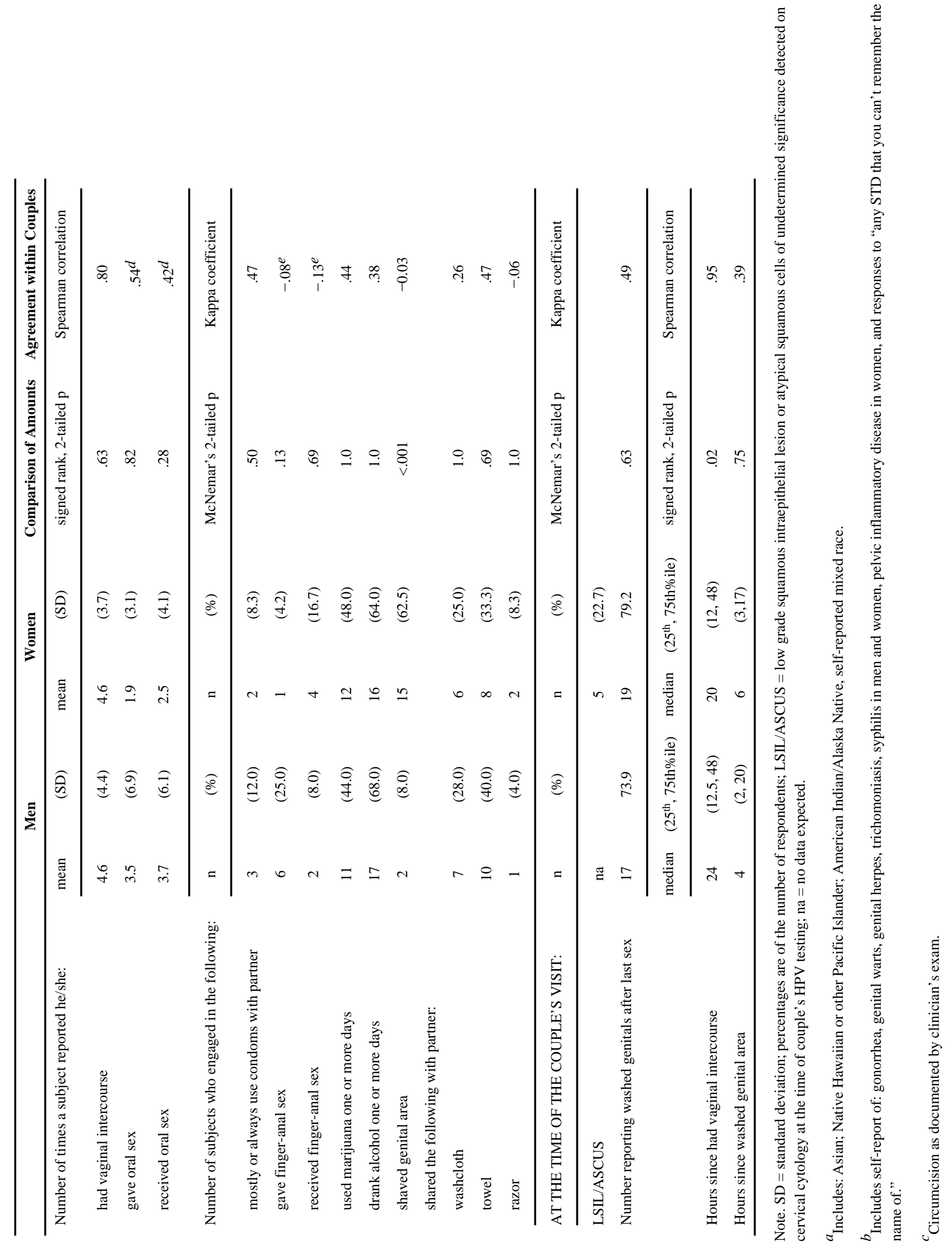




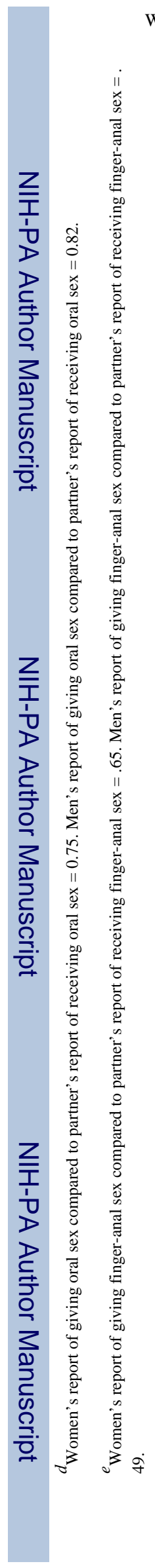

Widdice et al.

Page 12

J Adolesc Health. Author manuscript; available in PMC 2011 August 1. 
Table 2

HPV types detected in each partner from anogenital, palmar and oral areas during couple's visit and incident HPV types detected in women prior to couple's visit

\begin{tabular}{|c|c|c|c|c|}
\hline \multirow[t]{2}{*}{ Couple } & \multirow{2}{*}{$\begin{array}{l}\text { Incident HPV Type } \\
\text { CVL }\end{array}$} & \multicolumn{3}{|c|}{ Anatomical Area Sampled During Couple's Visit } \\
\hline & & Anogenital & Palmar & Oral \\
\hline A Male & na & - & - & - \\
\hline A Female & 405359 & - & - & - \\
\hline B Male & na & - & - & - \\
\hline B Female & 3162 & - & - & - \\
\hline C Male & na & - & - & - \\
\hline C Female & 16 & - & - & - \\
\hline D Male & na & 84 & 84 & - \\
\hline D Female & 83 & 84 & 5966 & - \\
\hline E Male & na & 16 & 16 & - \\
\hline E Female & 16 & 16 & 16 & - \\
\hline F Male & na & 83 & - & - \\
\hline F Female & 61 & 66183 & - & - \\
\hline G Male & na & 3962 & 62 & - \\
\hline G Female & 39 & 3916 & - & - \\
\hline H Male & na & 355262 & - & - \\
\hline H Female & 62 & 5462 & 355961 & - \\
\hline I Male & na & 52 & 6 & - \\
\hline I Female & 6 & 652 & - & - \\
\hline J Male & na & 167384 & 7384 & - \\
\hline J Female & 7384 & 7384 & 7384 & - \\
\hline K Male & na & 5152 & 5152 & - \\
\hline K Female & 51 & 5152 & 51 & - \\
\hline L Male & na & 16314553626684 & CP6108 163173 & - \\
\hline L Female & 316284 & 31545562 & - & 16 \\
\hline M Male & na & 3984 & 16 & - \\
\hline M Female & 3984 & 3984 & 3984 & - \\
\hline N Male & na & 518267 & - & - \\
\hline N Female & 51 & 518267 & 51 & - \\
\hline O Male & na & 535558705962 & - & - \\
\hline O Female & 525358 & 18525355586770 & - & IA \\
\hline P Male & na & 5884521639425367 & 163942515253588467 CP6108 & - \\
\hline P Female & 53684284 & 588439425152 & 5884 & - \\
\hline Q Male & na & 70188453 & 188470 & 16 \\
\hline Q Female & 7084 & 701884 & - & 16 \\
\hline R Male & na & - & - & - \\
\hline R Female & 515258 & 61 & - & - \\
\hline S Male & na & - & - & - \\
\hline S Female & 59 & 54 & - & - \\
\hline
\end{tabular}




\begin{tabular}{llllll}
\hline Couple & Incident HPV Type & & Anatomical Area Sampled During Couple's Visit & Oral \\
\cline { 2 - 5 } & CVL & Anogenital & Palmar & - \\
\hline T Male & na & - & 5354 CP6108 & - & - \\
T Female & 54 & 40 & - & - \\
U Male & na & - & - & - \\
U Female & 4045 & 61 & - & - \\
V Male & na & 1654 cp6108 & - & - \\
V Female & 16 & CP6108 & - & - \\
W Male & na & 16515361 & - & - \\
W Female & 16515361 & 5966 & - & - \\
X Male & na & 394251525358 & - & 39 \\
X Female & 39 & 1639 & - & - \\
Y Male & na & 5367 & - & - \\
Y Female & 53 & &
\end{tabular}

$\mathrm{CVL}=$ cervico-vaginal lavage; $\mathrm{NA}=$ no result expected for this subject; $-=$ negative for HPV-DNA; IA - human $\beta$-globin negative and HPV DNA negative sample. 


\section{Table 3}

Number and Percent of Couples with Type-Specific Anogenital Concordance.

\begin{tabular}{lll}
\hline Concordance Criteria & Number of Couples & Percent of All Couples \\
\hline Negative concordance & 3 & 12 \\
Positive concordance, number of types & & \\
1 & 6 & 24 \\
2 & 4 & 16 \\
3 & 2 & 8 \\
4 & 1 & 4 \\
5 & 1 & 4 \\
\hline Total & 17 & 68 \\
\hline
\end{tabular}




\section{Table 4}

Hygienic Characteristics and Sexual Behaviors Associated with Anogenital-Type-Specific Concordance at a pvalue $=<.10$ as Reported by Female and Male Partners.

\begin{tabular}{|l|c|c|c|}
\hline REPORTED HYGIENIC CHARACTERISTICS OR SEXUAL BEHAVIORS & Exact OR & $\mathbf{( 9 5 \%}$ Confidence Limits) & p-value \\
\hline Female partner's cytology and self-reported history and behaviors: & & & \\
\hline LSIL/ASCUS at time of couple's visit & 5.100 & $(.804$, Infinity) & 0.08 \\
\hline Shared towel with partner in past week & .153 & $(.019,1.017)$ & .04 \\
\hline Received finger-anal sex & .124 & $(.004,1.418)$ & .05 \\
\hline Shared razor with partner in past week & .186 & $(0,1.639)$ & .05 \\
\hline Lifetime history of genital warts & 3.94 & $(.628$, Infinity $)$ & .08 \\
\hline Washed genitals after sex & .235 & $(0,1.476)$ & .08 \\
\hline Hours since had vaginal intercourse & .980 & $(.953,1.000)$ & .03 \\
\hline Male partner's report of his own history and behaviors: & & & \\
\hline More than 10 lifetime opposite-sex partners & .141 & $(.005,1.249)$ & .05 \\
\hline Hours since had vaginal intercourse & .983 & $(.959, .999)$ & .02 \\
\hline Washed genital area after sex & .162 & $(0, .996)$ & .03 \\
\hline
\end{tabular}

Note. Exact OR = exact odds ratio from SAS Proc Logistic.

95\% Confidence Limits $=$ mid-p confidence limits; mid-p significance tests have the possibility of attaining a two-tailed value less than .05 while the OR's exact $95 \%$ confidence interval (CI) includes 1 (see text).

$\mathrm{p}$-value $=$ mid $\mathrm{p}$-value from conditional exact test.

LSIL/ASCUS = low grade squamous intraepithelial lesion or atypical squamous cells of undetermined significance detected on cervical cytology at the time of couple's HPV testing. 